\title{
Design of the passive joints of underactuated modular soft hands for fingertip trajectory tracking
}

This is the peer reviewed version of the following article:

Original:

Salvietti, G., Hussain, I., Malvezzi, M., Prattichizzo, D. (2017). Design of the passive joints of underactuated modular soft hands for fingertip trajectory tracking. IEEE ROBOTICS AND AUTOMATION LETTERS, 2(4), 2008-2015 [10.1109/LRA.2017.2718099].

Availability:

This version is availablehttp://hdl.handle.net/11365/1012985

since 2019-05-07T16:55:41Z

\section{Published:}

DOI:10.1109/LRA.2017.2718099

Terms of use:

Open Access

The terms and conditions for the reuse of this version of the manuscript are specified in the publishing policy. Works made available under a Creative Commons license can be used according to the terms and conditions of said license.

For all terms of use and more information see the publisher's website.

(Article begins on next page) 


\title{
Design of the Passive Joints of Underactuated Modular Soft Hands for Fingertip Trajectory Tracking
}

\author{
Gionata Salvietti $^{1,2}$, Irfan Hussain $^{1}$, Monica Malvezzi ${ }^{1,2}$ and Domenico Prattichizzo ${ }^{1,2}$
}

\begin{abstract}
In this work, we propose a method to design tendondriven underactuated hands whose fingertips can track a predefined trajectory, when actuated. We focus on passively compliant hands composed of deformable joints and rigid links. We firstly introduce a procedure to determine suitable joints stiffness and tendon routing, then a possible realization of a robotic underactuated finger is shown. The kinematic and kinetostatic analysis of a tendon-driven robotic finger is necessary to define the overall stiffness values of the finger joints. A structural analysis of the element constituting each passive joint allowed to define a relation between the stiffness and joint's main dimensional and material properties. We validated the proposed framework both in simulation and with experiments using the robotic SoftSixthFinger as a case study. The Soft-SixthFinger is a wearable robot for grasping compensation in patients with a paretic hand. We demonstrated that different fingertip trajectories can be achieved when joint stiffness and tendon routing are properly designed. Moreover we demonstrated that the device is able to grasp a wider set of objects when a specific finger flexion trajectory is designed. The proposed framework is general and can be applied to robotic hands with an arbitrary number of fingers and joints per finger. The modular approach furthermore allows the user to easily customize the hand according to specific tasks or trajectories.
\end{abstract}

Index Terms-Grippers and Other End-Effectors, Tendon/Wire Mechanism, Compliant Joint/Mechanism

\section{INTRODUCTION}

$\mathbf{R}$ OBOTIC grasping and manipulation require an elevated level of complexity which involves not only the mechanical structures of the end effectors, but also their control and coordination. Very sophisticated hands with a high number of degrees of freedom have been developed with the main aim of resembling the kinematic structure of the human hand. However, these complex devices resulted poorly capable to interact with objects and with the environment to fulfill a proposed task. In addition, the presence of many actuators increases the costs, decreases the robustness of the devices and demands for complex control systems able to manage the high number of control inputs.

This paper was recommended for publication by Editor Han Ding upon evaluation of the Associate Editor and Reviewers' comments. The research leading to these results has received funding from the EU Horizon 2020/2015 project No 645599 "SoMa" and from EU Horizon 2020/2015 project No 688857 "SOFTPRO".

${ }^{1}$ Università degli Studi di Siena, Dipartimento di Ingegneria dell'Informazione, Via Roma 56, 53100 Siena, Italy. [salviettigio, malvezzi, prattichizzo] ddiism.unisi.it

${ }^{2}$ Department of Advanced Robotics, Istituto Italiano di Tecnologia, Via Morego 30, 16163 Genoa, Italy.

Digital Object Identifier (DOI): see top of this page.
To deal with the above mentioned issues, a novel generation of underactuated compliant hands is growing in the robotic community in the last years, upgrading the preliminary attempts pioneered by Hirose et al., at the end of seventies [1]. Differently from classical multi-fingered hands, in these soft hands the interaction with the environment and the simplification of the control is a central aspect in the design of the device. The two pillars upon which these novel devices are built are the reduction of the degrees of actuation with respect to the total degrees of freedom (DoFs) and the intrinsic passive compliance at joint level. Underactuated hands have desirable adaptability to shapes, and can be effectively implemented using relatively simple differential and elastic elements [2]. The differential transmission solutions allow motion of other joints to continue after contact occurs on a coupled link, so that the hands can passively adapt to the grasped object shape [3]. Passive adaptability allows to drive the device with a reduced number of control parameters. Reasons for adding passive elements are manifold, including storing elastic energy, avoiding tendon slackness, distribution of forces over a large contact area, and overcoming the indeterminacy of configuration problem typical of an underconstrained mechanical system [4]. Shape adaptation increases the grasp performance by compensating the uncertainties in sensing, actuation and helps in stabilizing the grasp [5]. Most of the works in the literature focus on the development of new devices proposing different solutions in terms of actuation and materials for hand realization. Fewer works are instead focusing on systematic ways to improve the dexterity of new soft hands [6]. In a highly underactuated soft hand (e.g., a five fingered hand with 15 DoFs and one motor with a tendon driven actuation) there are few parameters to be tuned in order to design a desired motion for the robotic fingers. If the kinematic and the actuation system is fixed, a possible way to design a desired fingertip trajectory consists in tuning the stiffness of the passively compliant joints.

In this work, we present a systematic procedure to compute the stiffness ratio between the passive compliant joints so to obtain a desired trajectory for the fingertips. The proposed method assumes a given target motion of the fingertips and a given maximum actuation force for the tendon driven system to compute the stiffness value of the passive joints. It also assumes that the finger joints can be realized with a specific stiffness and pre-form shapes such that a single-cable actuation can be used. Furthermore, we propose a modular approach to define robotic hands. In most of the solutions existing in the 


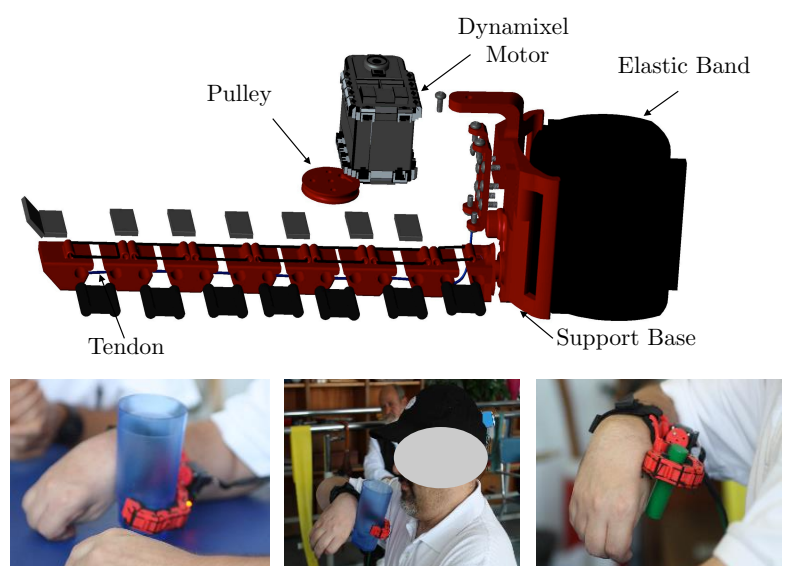

Fig. 1. The Soft-SixthFinger. The CAD exploded view of complete SoftSixthFinger together with its possible applications for grasp compensation.

literature, the hand is composed of a series of identical fingers, and the modularity is exploited at the finger level, e.g., see [7]. In the approach we propose, each phalanx, composed of a flexible and a stiff part, is a module. Beside the number and position of fingers in the hand, we can furthermore change the number of phalanges and some of their structural properties, similarly to the approach proposed in [8]. The problem that we want to solve can be formulated as follows: how can we design finger joint stiffness so that, when applying a certain force to the tendons, the joint configuration vector $\mathbf{q}$ follows a desired value $\mathbf{q}_{r}(t)$ over time $t$, so that the fingertip follows a desired trajectory $\mathbf{p}_{r}(t)$ ? To address this problem, we propose a method to compute the stiffness given a certain kinematics of the robotic hand. We then exploit the beam theory to find a way to compute the stiffness for a particular geometry of the soft parts. Differently from [9] where generic motion is obtained designing different planar linkages, we adopted a simple serial kinematic chain, in which we can choose the number of elements. We focus on trajectories more common on power grasp executed by robotic hands, that can be obtained considering a deformation only at joint level. We present the application of this approach in a case study represented by the Soft-SixthFinger [10], see Fig. 1. We choose this particular device to demonstrate that the possibility of selecting the pre-shaping configuration and the finger flexion trajectory is important to enhance soft hands grasping capabilities [11].

\section{DESIGN OF FINGERTIP TRAJECTORY}

In this section, we propose a mathematical framework to compute the stiffness of compliant joints necessary to track a desired trajectory with the fingertips of a compliant underactuated hand. In particular, we define how to compute the stiffness values related to a given trajectory, and then how to realize passive compliant joints with a given stiffness once the geometry of the passive joints is defined. Finally, we propose how the computed stiffness values are related to tendon routing throughout the finger. We investigated this problem since fingertip trajectories play an important role in robotics and human grasping [11], [12]. In particular, in robotic hands the trajectories of the fingertips can be planned according to a specific task or to the shape of the object to grasp [13]. Another interesting approach to generate trajectories from mapping procedures is that described in [14], where postural synergies identified for the human hand are replicated on robotic hands with dissimilar kinematic structures, allowing to define a middleware control interface that is device-independent.

\section{A. Problem definition}

Let us assume a hand composed of $n_{f}$ fingers, with $n_{q}$ joints, actuated by a series of $n_{t}$ tendons. For the sake of simplicity, we assume that all the joints in the hand are revolute (R), so that the variable $q_{i}$ describing $i$-th displacement is a rotation. Let us indicate with $\mathbf{q}=\left[q_{1}, \cdot, q_{n_{q}}\right]^{\mathrm{T}} \in \Re^{n_{q}}$ a vector containing hand joint rotations and with $\mathbf{t} \in \Re^{n_{t}}$ tendon displacements. Let us collect in the vector $\mathbf{p} \in \Re^{n_{f} n_{d}}$ the vectors $\mathbf{p}_{i} \in \Re^{n_{d}}$ describing position (and, in case, orientation) of a reference frame representing the $i$-th fingertip with respect to a reference frame that we assume as a basis, fixed, for instance, on hand palm. We indicate with $n_{d}$ the dimension of the configuration space for the fingertip, for example, $n_{d}=3$ in the planar case, $n_{d}=6$ in the more general three dimensional case.

From the kinematic analysis of hand fingers, it is possible to relate tendon displacements $\mathbf{t}$ to hand joint configuration $\mathbf{q}$, i.e.,

$$
\mathbf{t}=\mathbf{t}(\mathbf{q})
$$

where $\mathbf{t}(\mathbf{q}): \Re^{n_{q}} \rightarrow \Re^{n_{t}}$ is the tendon forward kinematics function, that in the more general case is nonlinear and depends on system geometric dimensions and tendon routings. Considering a small variation of tendon lengths and joint angles with respect to a reference configuration, the above relationship can be linearised as

$$
\delta \mathbf{t}=\mathbf{T}(\mathbf{q}) \delta \mathbf{q}
$$

where $\mathbf{T}(\mathbf{q})$ is the Jacobian of the function introduced in eq. (1), that can be evaluated as $\mathbf{T}(\mathbf{q})=\frac{\partial \mathbf{t}}{\partial \mathbf{q}}$. In some cases, when the tendons are connected through pulleys with a circular radius, it can be shown that eq. (1) is linear and then $\mathbf{T}$ matrix is constant. In this case $\mathbf{T} \in \Re^{n_{t} \times n_{q}}$ is a transformation matrix whose elements depend on finger pulleys' sizes and tendon routing topology and is independent from hand posture [2]. By applying the Principle of Virtual Work to the hand it is possible to obtain the dual static relationship

$$
\boldsymbol{\tau}_{a}=\mathbf{T}^{\mathrm{T}} \mathbf{f}
$$

where $\mathbf{f} \in \Re^{n_{t}}$ is a vector containing tendons' pulling forces, and $\tau_{a} \in \Re^{n_{q}}$ represents the corresponding hand joint torques. The following equation can be used to describe hand dynamics

$\mathbf{B}(\dot{\mathbf{q}}) \ddot{\mathbf{q}}+\mathbf{n}(\mathbf{q}, \dot{\mathbf{q}})=\boldsymbol{\tau}_{a}+\boldsymbol{\tau}_{f}(\mathbf{q}, \dot{\mathbf{q}})+\boldsymbol{\tau}_{g}(\mathbf{q})+\boldsymbol{\tau}_{p}(\Delta \mathbf{q})+\mathbf{J}^{\mathrm{T}} \mathbf{f}_{c}$,

where $\tau_{p}(\Delta \mathbf{q})$ expresses the torque generated by the deformation of elastic elements, $\boldsymbol{\tau}_{f}(\mathbf{q}, \dot{\mathbf{q}})$ expresses the equivalent joint torques due to frictional effects, $\boldsymbol{\tau}_{g}(\mathbf{q})$ models the gravitational terms, $\mathbf{B}(\dot{\mathbf{q}})$ represents the inertia matrix, $\mathbf{n}(\mathbf{q}, \dot{\mathbf{q}})$ contains centrifugal and Coriolis terms, $\mathbf{J}^{\mathrm{T}} \in \Re^{n_{q} \times n_{c}}$ is 
the transpose of finger Jacobian matrix and $\mathbf{f}_{c} \in \Re^{n_{c}}$ is a vector containing the interaction forces that the environment or a grasped object apply to the fingers, whose dimension $n_{c}$ depends on the number and type of contacts [15]. $\boldsymbol{\tau}_{p}(\Delta \mathbf{q})$ can be evaluated as a function of joint deformation $\Delta \mathbf{q}$, evaluated w.r.t. a reference (rest) position of the hand $\mathbf{q}_{0}$, i.e., $\Delta \mathbf{q}=\mathbf{q}-\mathbf{q}_{0}$. Typically underactuated and compliant hands have quite slow velocities and accelerations, furthermore they are realized with light materials and have a limited number of actuators, so that we can neglect the inertial and viscous terms. Furthermore, we assume that the torque generated by the deformation of elastic elements is higher than the one due to gravitational effects. The friction between tendons and finger parts could give a not negligible contribution, however its prediction depends on several parameters that cannot be easily considered (e.g., surface and material properties). Assuming a simplified friction model characterized by the friction static coefficient $\mu_{s}$, we can suppose that, at each joint $i$, friction between the tendon $j$ and the links causes a reduction of tendon tension $\Delta f_{f, j, i}$ that can be approximated as $\Delta f_{f, j, i}=f_{r, j} \sum_{k=1}^{i} \frac{e^{\mu_{s} q_{k}-1}}{e^{\mu_{s} q_{k}}}$, and that the corresponding joint torque due to friction can be evaluated as

$$
\tau_{f, i}=-f_{r, j} h \sum_{k=1}^{i} \frac{e^{\mu_{s} q_{k}}-1}{e^{\mu_{s} q_{k}}},
$$

where $h$ is the distance between the elastic element bending axis and the tendon when the joint is in its rest position and $f_{r, j}$ is the force applied to the tendon $j$. It is evident that the torque generated by friction depends on joint configuration, on the reference tendon force and on material properties. To the best of our knowledge, reliable data on frictional properties of the materials that we employed to build the prototype described in this work are not easily available in the literature, since this type of materials and manufacturing processes are quite recent and still under investigation. In this first study on the design of passive joints, we did not consider friction contribution in our evaluation. Further developments of this study will include a more detailed discussion on this aspect. Finally, if we consider a free motion in which the finger does not interact with external objects or surfaces, we can assume $\mathbf{f}_{c}=\mathbf{0}$. With the above simplifications, the dynamics of the system can be reduced to

$$
\boldsymbol{\tau}_{a}+\boldsymbol{\tau}_{p}(\Delta \mathbf{q})=0
$$

Also this expression can be linearised: if we consider a variation of the loading condition with respect to an initial reference configuration, the variation of torque generated by joint deformation can be evaluated in this case as $\delta \tau_{p}=-\mathbf{K}_{q} \delta \mathbf{q}$, so that the dynamics of the system can be approximated as

$$
\delta \boldsymbol{\tau}_{a}=\mathbf{K}_{q}(\mathbf{q}) \delta \mathbf{q} .
$$

The linearisation leads to the introduction of the joint stiffness matrix $\mathbf{K}_{q} \in \Re^{n_{q} \times n_{q}}$, symmetric and positive definite, that can be evaluated, at a given configuration $\mathbf{q}$, as $\mathbf{K}_{q}=-\frac{\partial \boldsymbol{\tau}_{p}}{\partial \mathbf{q}}$. In the more general case, stiffness matrix $\mathbf{K}_{q}$ depends on passive joint structural properties, namely geometrical dimensions and material parameters, but also on joint configuration.
In this framework, we can reformulate the question proposed in the introduction as: how can we design hand joint stiffness matrix $\mathbf{K}_{q}$ so that, when applying a certain force profile $\mathbf{f}_{r}(t)$ to the tendons, the fingertips of the hand follow a certain trajectory $\mathbf{p}_{r}(t)$ ? To solve this problem, we sampled the desired trajectory $\mathbf{p}_{r}(t)$ in a series of elementary steps and we solved for each step the linearised equilibrium equation (7). The first step consists in solving the inverse kinematics for each finger, finding, for each time sample $t_{k}$ (the subscript $k$ indicates the generic time sample) the joint configuration vector $\mathbf{q}_{r}\left(t_{k}\right)$ corresponding to the desired fingertip configuration $\mathbf{p}_{r}\left(t_{k}\right)$. The variation of hand configuration corresponding to the sample $k$ is given by $\delta \mathbf{q}_{k}=\mathbf{q}_{r}\left(t_{k}\right)-\mathbf{q}_{r}\left(t_{k-1}\right)$. This variation is produced by applying to the tendons a force variation $\delta \mathbf{f}_{k}=\mathbf{f}_{r}\left(t_{k}\right)-\mathbf{f}_{r}\left(t_{k-1}\right)$.

The next step consists in evaluating the stiffness matrix that allows to replicate that elementary motion. Assuming that the $\mathbf{K}_{q}$ matrix is diagonal (i.e., the joints are independent), the problem can be straightforwardly solved: eq. (4) can be rewritten, in this case, as

$$
\delta \boldsymbol{\tau}_{k}=\mathbf{Q}_{k} \mathbf{k}_{q_{k}}
$$

where $\mathbf{Q}_{k} \in \Re^{n_{q} \times n_{q}}$ is defined as $\mathbf{Q}_{\mathbf{k}}=\operatorname{diag}\left(\delta \mathbf{q}_{k}\right)$, while $\mathbf{k}_{q_{k}} \in \Re^{n_{q}}$ is a vector collecting joint stiffness values contained in $\mathbf{K}_{q}$ matrix principal diagonal at time sample $k$. Taking into account eq. (3), the system can be solved as

$$
\mathbf{k}_{q_{k}}=\mathbf{Q}_{k}^{-1} \mathbf{T}_{k}^{\mathrm{T}} \delta \mathbf{f}_{k},
$$

where $\mathbf{T}_{k}$ is the tendon Jacobian matrix evaluated for the joint configuration vector $\mathbf{q}_{k}$. The solution of the linear system in eq. (9) is a vector containing hand joint stiffness values at sample $k$ that allow to obtain a configuration variation $\delta \mathbf{q}_{k}$ of the hand when the tendons are pulled with a force variation $\delta \mathbf{f}_{k}$.

Remark 1: It is worth to observe that the stiffness values depend on both joint displacement $\mathbf{q}$ and on tendon force $\mathbf{f}_{r}$. If we change the overall applied force to $\alpha \mathbf{f}_{r}$, where $\alpha>0$ is a generic scaling factor, the same joint configuration can be followed by scaling the stiffness matrix with the same scaling factor, i.e., with a stiffness matrix $\alpha \mathbf{K}_{q}$. In other words, the shape of fingertip trajectories does not depends on $\mathbf{K}_{q}$ absolute values, but rather on their relative ratios. Normalizing vector $\mathbf{k}_{q}$ we obtain a base for the subspace of possible stiffness combination that can be used to track a desired trajectory. The absolute values of $\mathbf{K}_{q}$ can be set on the basis of material properties and geometry constraints in the design of hand fingers, as we will better detail in Sec. III.

Remark 2: The procedure described so far leads, in the general case, to a stiffness matrix that depends on hand configuration, i.e., $\mathbf{K}_{q}=\mathbf{K}_{q}(\mathbf{q})$. The design of hand joints to obtain a desired stiffness value may be not simple if the dependency of $\mathbf{K}_{q}$ on $\mathbf{q}$ is significant. In Section III, we will show two applications in which we obtain stiffness values that slightly depends on hand configuration and in which we could consider their values constant with respect to $\mathbf{q}$.

Remark 3: In some cases the procedure previously described leads to negative stiffness values for some joints. This result practically means that the sign of rotation angle for that 
joint is opposite to the one that was considered positive in the representation of finger kinematics. This change of sign can be managed by changing tendon routes in the rigid elements adjacent to the flexible joint that presents the stiffness value. In Section III we will present an example in which, by applying the proposed procedure to a particular trajectory, we get a negative value for the stiffness of the proximal joint.

\section{B. Modular element modeling}

Let us assume that a generic finger, indicated with index $j$, is realized by connecting $n_{q_{j}}$ modules with the same geometric dimensions, but with different stiffness values. Let us assume, for the sake of simplicity, that each finger is actuated by a single tendon, let us indicate with $t_{j}$ its length variation. The analysis can be easily extended to hands actuated with multiple tendons. Let us indicate with $l$ the length of the elastic element that constitute the joint. According to the modular approach proposed in this paper, we assume that this value is the same for all the joints, however the analysis can be easily extended to hands with different joints.

Let us then consider a generic joint $i$ on the finger. The main geometrical parameters adopted in this section are summarized in Fig. 2. When the tendon is pulled by a force $f_{r, j}$ the elastic elements in the joints bend: let us suppose that the joint $i$ is rotated by an angle $q_{i}=\theta_{i}$, the corresponding variation of tendon length in the joint is indicated with $\Delta l_{i}$. We suppose that the bending elastic element assumes a circular arc shape, with radius $r_{i}=\frac{l}{q_{i}}$, and we indicate with $a_{i}$ the arc chord, that can be evaluated as $a_{i}=2 r_{i} \sin \frac{q_{i}}{2}$. The corresponding variation of tendon length can be evaluated as

$$
\Delta l_{i}=l-\frac{a_{i}}{r_{i}}\left(r_{i}-h\right)=l-2 r_{i} \sin \frac{q_{i}}{2}\left(\frac{r_{i}-h}{r_{i}}\right) .
$$

The overall tendon displacement can be expressed as the sum of all the variations over finger joints, i.e., $t_{j}=\sum_{i=1}^{n_{q_{j}}} \Delta l_{i}$. We can observe that therefore each component of eq. (1), namely $t_{j}=t_{j}(\mathbf{q})$, is nonlinear. The $(j, i)$-th element of the Jacobian matrix $\mathbf{T}$ introduced in eq. (2) can be evaluated as

$$
T_{j, i}=\frac{\partial t_{j}}{\partial q_{i}}=-\cos \left(\frac{q_{i}}{2}\right)\left(\frac{l}{q_{i}}-h\right)+2 \frac{l_{i}}{q_{i}^{2}} \sin \left(\frac{q_{i}}{2}\right),
$$

and is dependent on joint configuration. However, for small values of $q_{i}$, we can approximate $\left(\sin \frac{q_{i}}{2}\right) \approx \frac{q_{i}}{2}$, obtaining

$$
\Delta l_{i} \approx h \theta_{i}, T_{j, i} \approx h .
$$

For $h=6 \mathrm{~mm}$ and $l=15 \mathrm{~mm}$, the approximations in eq. (12) lead to an error on $T_{j, i}$ values within $9 \%$ for $q_{i}<0.8 \mathrm{rad}$.

\section{Passive joint design}

In this subsection, we focus on hardware realization of soft joints with a given stiffness. Our approach is inspired to the work presented in [16] for soft grippers composed of flexible and stiff parts. The model takes into consideration the geometric and material properties of the robotic device. The realization of soft grippers using closed-top molding techniques is further detailed in an open source platform named Soft Robotics Toolkit [17]. Differently from that work,

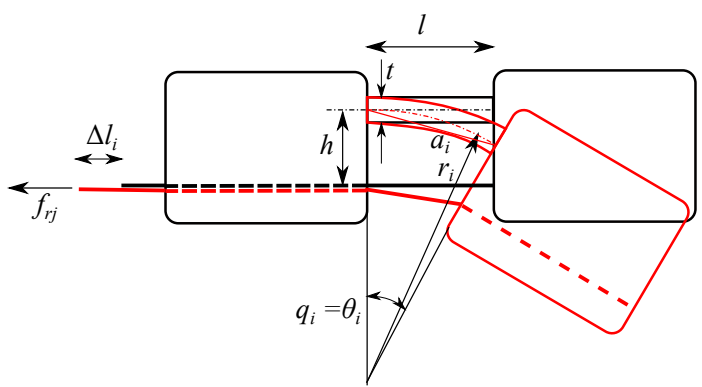

Fig. 2. Main geometrical parameters of the modular passive joint.

we exploited mechanical properties of flexible materials and their dependency on fabrication methods so to obtain different stiffness values of flexible parts through the variation of the percentage of infill density, that can be regulated during the $3 \mathrm{D}$ printing phase.

In this paper, we furthermore follow a modular approach in the design. The basic idea is to realize modules composed of a flexible passive joint, in which the stiffness can be varied by properly regulating printing parameters, and a rigid link. The rigid link is designed in order to allow, if necessary, the switch in tendon routing necessary to invert the sign of torque generated by tendon actuation force and then manage negative $k_{q, i}$ values. Modules can be then combined together to build underactuated soft robotic fingers able to track desired fingertip trajectories. Using a sufficiently high number of modules, the deflection of each joint while the finger is bending is rather low, so that, for each joint, we can use the simplified linear results of beam theory. For higher deflections, a more complete bending model, as the one described in [18] should be adopted. When the actuator applies a force $f_{r, j}$ through the $j$-th tendon, it produces on the joint $i$ a torque $\tau_{i}=f_{r, j} h$. The corresponding joint rotation angle can be evaluated as $\theta_{i}=\frac{f_{r, j} h l}{E_{i} I_{i}}$, where $E_{i}$ is the Young's modulus of the material, $I_{i}$ is the second moment of area, and $l$ is the length of the module elastic part. The rotational stiffness of the joint can be then evaluated as

$$
k_{q, i}=\frac{E_{i} I_{i}}{l} .
$$

Assuming that the flexible part of the module has a rectangular cross section, the second moment of area is $I_{i}=\frac{w_{i} t_{i}^{3}}{12}$. Referring to eq. (13), we can observe that different joints stiffness can be achieved by changing either the geometric or material parameters of the modules. Since we aim at realising a modular structure, we assume that the geometric parameters are the same for each module, i.e. $I_{i}=I, w_{i}=w, t_{i}=t$. On the other hand, we can tune the material parameters, specifically $E_{i}$, in order to vary the overall stiffness of the modules.

\section{CASe Study: The Soft-SixthFinger}

The mathematical framework presented in Sec. II can be applied to a wide range of robotic grippers. In this section, we propose as a case study the Soft-SixthFinger. The Soft- 


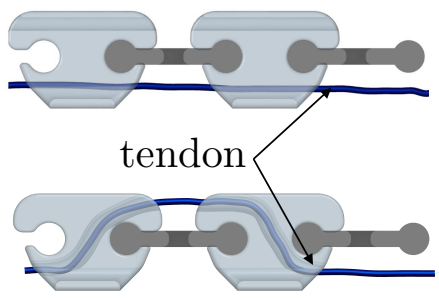

Fig. 3. The two possible configurations of tendon routing. On top, the straight while on bottom, the switched configuration is shown.

SixthFinger is a device designed to be used to compensate the missing grasping abilities of chronic stroke patients [10].

The exploded view of the device and examples of its applications have been previously introduced in Fig. 1. The working principle of the Soft-SixthFinger is to replicate the two parts of a simple gripper using on one side the paretic forearm of a patient and, on the other side, a flexible finger that can be worn at the wrist with the help of an elastic band. We use this device as case study for two main reasons. Firstly, in earlier version of the device [10], the trajectory of the finger was regulated manually by setting the joints stiffness by hit and trial methods. The mathematical model proposed in this work allows to obtain suitable stiffness at each joint which improved not only the grasping performance of the device but also considerably reduces the manufacturing iterations. Secondly, the proposed method can be validated on a finger base. In fact, the idea is that a fingertip is able to track a certain trajectory when selecting the appropriate joint stiffness and tendon routing. In case of multi-fingered hands the process is simply replicated for each finger.

The Soft-SixthFinger is built with a modular structure. Each module is composed of a rigid part realized in ABS (Acrylonitrile Butadiene Styrene, ABSPlus, Stratasys, USA) and a thermoplastic polyurethane part (Lulzbot, USA) that acts as the flexible joint. We selected polyurethane for flexible parts because the high elongation of this material allows for repeated movement and impact without wear or cracking proving also an excellent vibration reduction. We considered seven modules in order to achieve a length of the finger similar to the average size of human hand [10]. Fig. 3 shows the two possible configurations of tendon routing, we named as straight and switched configuration. The desired trajectory of the finger can be obtained by tuning the joints' stiffness and selecting the suitable tendon configuration.

\section{A. Finger module stiffness evaluation}

We analyzed the possibility of tuning finger joint stiffness values exploiting the potentialities of $3 \mathrm{D}$ printing fabrication methods. In particular, choosing the thermoplastic polyurethane as material for realizing the flexible parts, we can get different stiffness values, while maintaining the same geometric shape, by regulating the percentage of infill density. This parameter affects primarily material density, but also its mechanical properties. The first two columns of Table I summarizes the variation of Young's modulus $E$ of the thermoplastic polyurethane as a function of the infill percentage

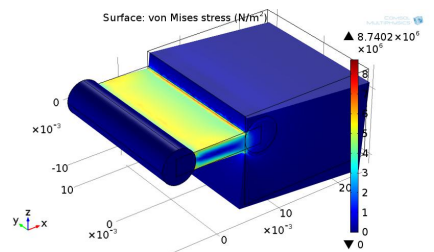

a)

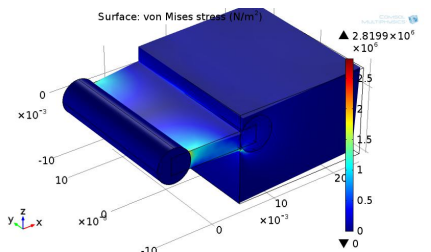

c)

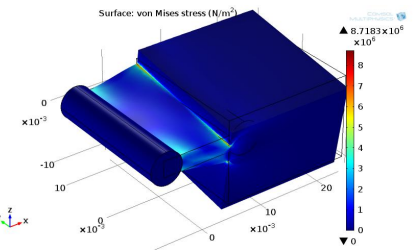

e)

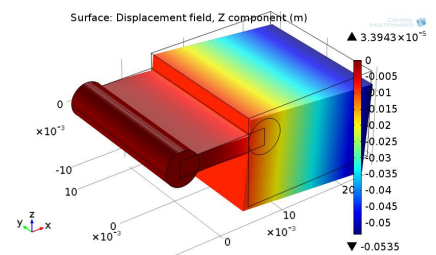

b)

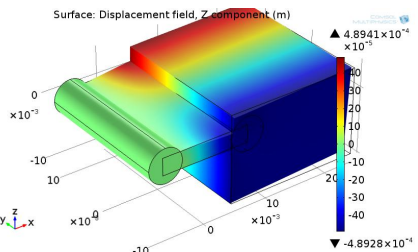

d)

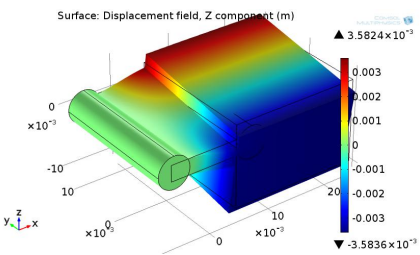

f)
Fig. 4. FEM model and stress/deformation analysis of the passive joint. In all the simulations, the left part of the flexible link was constrained to be fixed, while a load was applied to the rigid module connected to the right. Different loading conditions were applied to estimate the equivalent stiffness in different directions. The diagrams reported here summarizes the results obtained for the $100 \%$ of infill density. For each row, the left diagram show stress distribution and the right one module deformation $a, b$ ): bending in the principal direction, a $5 \mathrm{~N}$ load was applied to the free boundary of the rigid module in the $z$ direction. $\mathrm{c}, \mathrm{d}$ ): bending in the lateral direction, a $5 \mathrm{~N}$ load was applied to the free boundary of the rigid module in the $y$ direction. $\mathrm{e}, \mathrm{f}$ ): torsion, a $\pm 5 \mathrm{~N}$ load was applied to the free lateral boundaries, so to produce an equivalent torsional moment of about $100 \mathrm{Nmm}$ with respect to the $x$ direction.

density $\rho$ [19]. Such a relation has been obtained from experimental tests. Another aspect that should be taken into account in this type of production is the material anisotropy, i.e. the dependency of mechanical properties on layer printing directions. Such an aspect may assume a significant weight in low cost additive manufacturing techniques as Fusion Deposition Modeling (FDM), while could be reduced using for example Stereolithography (STL) techniques. The prototypes described in this paper have been realized with a standard FDM process. Further investigations on material properties to realize passive joints in modular robotic fingers are necessary to fully exploit the potentialities of the proposed methodology and it is planned as future work [20].

For a given geometric structure of the joint, the corresponding stiffness value is therefore related to the infill density, i.e. $k_{q i}=f\left(\rho_{i}\right)$. We evaluated the stiffness of a modular element varying material properties using the Finite Element Method (FEM) based software COMSOL. The dimensions of the elements are: length $l=13 \mathrm{~mm}$, thickness $t=2.5 \mathrm{~mm}$, width $w=21 \mathrm{~mm}$. Fig. 4 and Table I summarizes the main obtained results, in terms of stress and deformation of each module, and joint stiffness for different values of infill density percentage. Another parameter that we need to consider is 
TABLE I

TPU MECHANICAL PROPERTIES (YOUNG MODULUS VARIATION) FROM [19], AND STIFFNESS VALUES EVALUATED WITH FEM ANALYSIS OF THE PASSIVE JOINT AS A FUNCTION OF INFILL DENSITY PERCENTAGE OF THE MATERIAL $\rho \%$, FOR A GIVEN GEOMETRY.

\begin{tabular}{|c|c|c|c|c|}
\hline$\rho \%$ & $\mathrm{E}(\mathrm{MPa})$ & $k(\rho) \mathrm{Nmm} / \mathrm{rad}$ & $k_{l} \mathrm{Nmm} / \mathrm{rad}$ & $k_{t} \mathrm{Nmm} / \mathrm{rad}$ \\
\hline \hline 10 & 1.07 & 4.6930 & 331.3 & 24.9 \\
\hline 30 & 1.38 & 6.0526 & 427.2 & 32.1 \\
\hline 50 & 2.07 & 9.0789 & 640.8 & 48.1 \\
\hline 70 & 6.53 & 28.6403 & 2021.6 & 151.7 \\
\hline 90 & 9.45 & 41.4474 & 2925.6 & 219.7 \\
\hline 100 & 10.50 & 46.052 & 3807.9 & 285.7 \\
\hline
\end{tabular}

the passive flexibility of the finger in the lateral direction and with respect to a torsional load, that cannot be controlled by actuators, and that could affect system functionality. We therefore evaluated also the stiffness of each module in the lateral direction, $k_{l}$, and the torsional stiffness, $k_{t}$. We reported the main results of this analysis in Fig. 4 and Tab. I. We can observe that, for the given geometry, the lateral stiffness is much higher than the bending one, due to the module geometry, while the torsional one is closer. A finger composed of simple modules as those considered in this analysis may therefore have an excessive torsional compliance, that however could be potentially compensated by modifying the design of the rigid part of the model.

\section{B. Trajectory computation}

To let the Soft-SixthFinger be able to grasp a wide range of objects, it is important to define suitable finger flexion trajectories. To this aim, we took inspiration from the motion of the human hand. In particular, we used the mapping algorithm originally proposed in [14], [21] to transfer the motion of the human hand onto robotic hands with dissimilar kinematics. The mapping method allows to compute the Soft-SixthFinger fingertip trajectories replicating the effects in terms of motions and deformations that a human reference hand would produce on a virtual object. This allows to work directly on the task space avoiding a specific projection between different kinematics. Details of the mapping are not reported here for the sake of brevity, interested readers can refer to [14], [21]. We simulated the motion of a human hand model according to the first and second synergy as defined in [22] using the SynGrasp Matlab toolbox [23]. We considered a model of a human hand augmented with a model of the robotic finger. We then computed the trajectories of the robotic fingertip using the above mentioned mapping algorithm. These target trajectories in the Cartesian space are therefore the result of the mapping of the first and second human hand synergies onto the robotic device.

We then adopted the procedure presented in Sec. II to evaluate the joints stiffness that allows to track a desired trajectories when applying a force to the tendon. Considering thermoplastic polyurethane as material for the flexible joints, we obtained the $\mathbf{k}_{q}$ values graphically reported in Fig. 5 and 6 for the first and second synergy, respectively. From the analysis of the results we firstly observed that the stiffness variation for each joint for the proposed trajectories is rather low. We then considered for each joint the mean $\mathbf{k}_{q}$ value. For instance, for the first synergy the mean stiffness values were

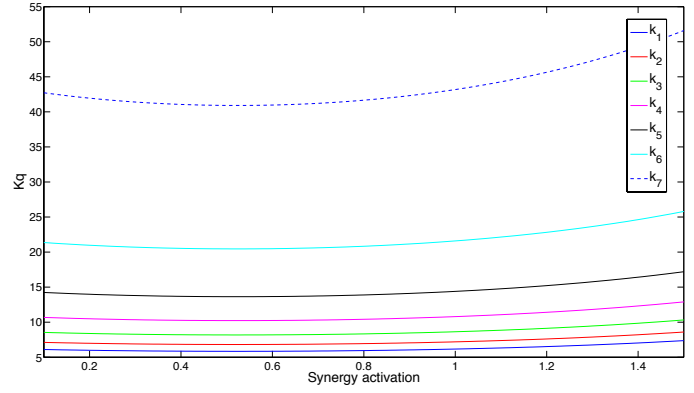

Fig. 5. Joint stiffness values necessary to generate the fingertip trajectory of the robotic sixth finger tip corresponding to the first synergy, evaluated according to Eq. (9), as a function of the corresponding actuation rate of the first synergy.

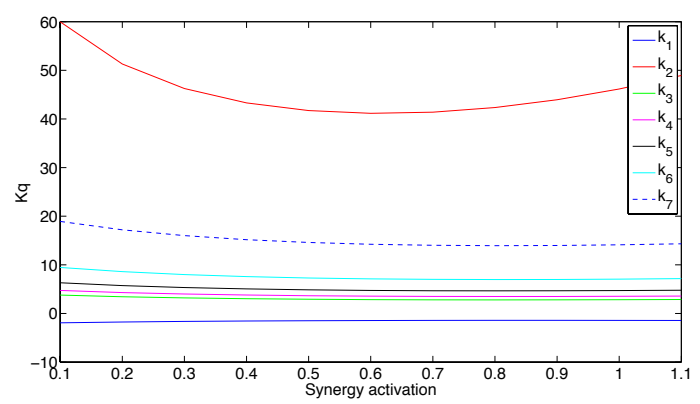

Fig. 6. Joint stiffness values necessary to replicate the second synergy trajectory of the robotic sixth finger tip, evaluated according to Eq. (9), as a function of the corresponding actuation rate of the second synergy. It can be noticed that the values obtained for the first joint are negative.

6.6 Nmm/rad, 7.7 Nmm/rad, 9.2 Nmm/rad, 11.5 Nmm/rad, 15.3 Nmm/rad, 23.1 Nmm/rad, 46.1 Nmm/rad from joint one to seven, respectively, which correspond to the following infill density percentage: $14.3,16.7,20,25,33.4,50,100$. The range of stiffness values for the joints was decided according to the properties of the material used for the soft joints. We selected the range of values that were possible to replicate using that material (NinjaFlex, Lulzbot, USA) and a 3D printer. For the second synergy, we can observe (Fig. 6) that the stiffness value for the first (proximal) joint is negative. For the first joint, we needed to adopt a different tendon routing, so to change the sign of the torque and consequently the rotation direction when the tendon is pulled, as shown in the second configuration of Fig. 3.

In the applications that we presented in this paper, fingertip trajectories were inspired by human hand first and second postural synergies, and they were quite simple and smooth. We therefore obtained for the joints smooth and approximately constant stiffness values, so that we could apply simple structural mechanic properties to design their passive elements. In a more general case stiffness values could significantly vary as a function of joint configuration. The design problem in this case would be more complex and would require further analysis, examples on how this problem can be faced can be found, for example in [9], [24].

\section{Experiments}

In order to experimentally validate our method, we realized three Soft-SixthFinger configurations with different stiffness 


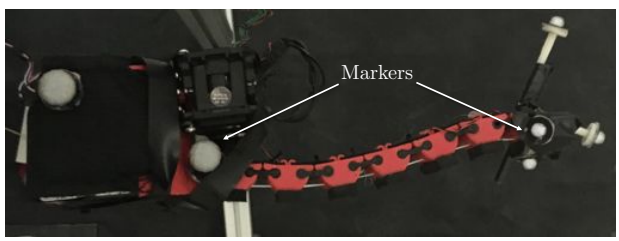

Fig. 7. The setup for fingertip tracking using the Vicon system.

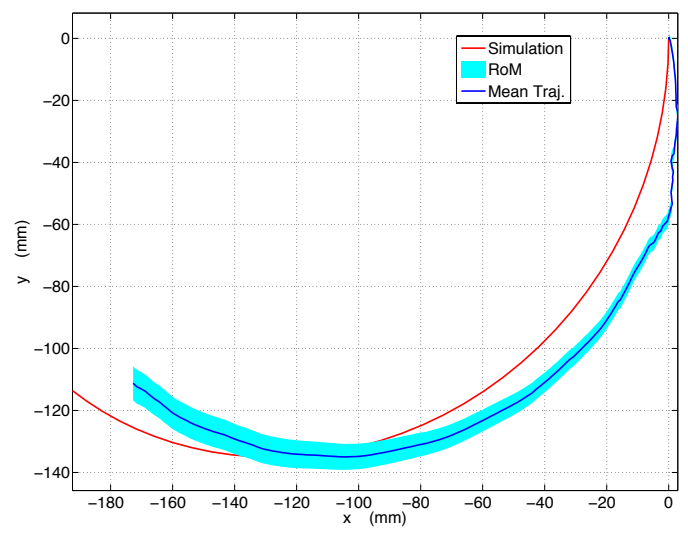

Fig. 8. Trajectory of the fingertip of the Soft-SixthFinger simulated (red) and experimentally evaluated (blue) for the first mapped synergy. The area in cyan represents the range of motion of the fingertip across ten trials.

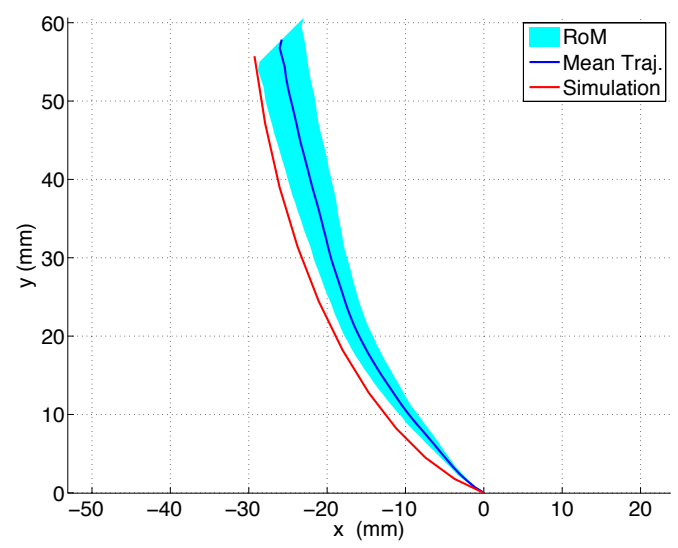

Fig. 9. Trajectory of the fingertip of the Soft-SixthFinger simulated (red) and experimentally evaluated (blue) for the second mapped synergy. The area in cyan represents the range of motion of the fingertip across ten trials.

at joint level and tendon routing. We printed the flexible parts with different percentage of infill density to achieve different deflection in joints under the same applied tendon force. Two configurations were realized using the computed infill density percentage, while for the third we used the same stiffness value for all the joints. We evaluated the fingertip trajectories of the proposed configurations. We used a Vicon system (Vicon Capture Systems, UK) consisting of 8 cameras to track the fingertip trajectories. Six passive optical markers were used as showed in Fig. 7. The computed and measured fingertip trajectories of the both configurations are reported in Fig. 8 and Fig. 9 for the first and second synergy, respectively. The area in cyan represents the range of motion of the fingertip across ten repeated motions. The blue line reports the mean trajectory between the ten measured. Note that the path length is shorter for the second synergy since a different starting configuration
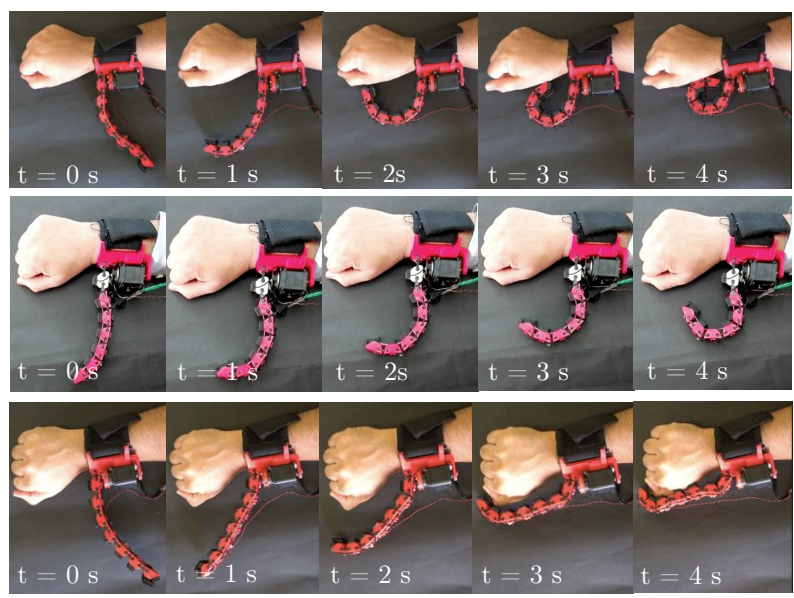

Fig. 10. Trajectories of the Soft-SixthFinger using different stiffeness at joint level. On top row the joint stiffness is computed so to track the first mapped synergy. In the central row, the joint stiffness is selected so to achieve the second mapped synergy. On the bottom row, the trajectory obtained considering all the joints with the same stiffness value.
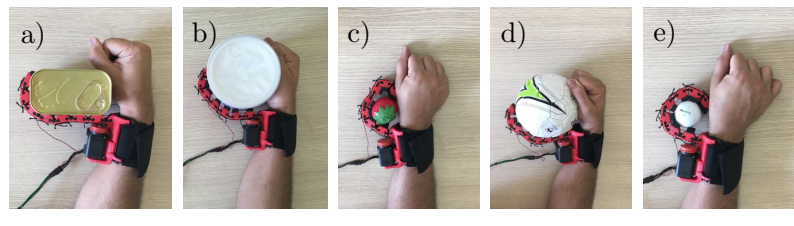

Fig. 11. The Soft-SixthFinger grasping objects with different shapes and sizes using the first mapped synergy.

is considered. The mean error between the simulated trajectory and the experimentally evaluated one for the first synergy was $0.023 \mathrm{~m}$ along $x$-axis and $0.021 \mathrm{~m}$ along $y$-axis, while for the second synergy it was $0.003 \mathrm{~m}$ along $x$-axis and $0.005 \mathrm{~m}$ along $y$-axis. This error is due to different factors including the 3D printed accuracy in density regulation, non-modeled friction between the tendon and the stiff part of the modules and small finger fluctuation during the flexion motion.

We performed a qualitative experiment in order to evaluate how the joints' stiffness regulate the trajectory of the finger and, consequently, how the flexion trajectory influence the device adaptability to different shapes of the grasped objects. A healthy subject was asked to wear the devices on its right arm, while simulating the paretic hand. Fig. 10 shows the snapshots of the three configurations of Soft-SixthFinger with different joints stiffness. The top and central row configurations have the stiffness in each joint computed using the design method introduced in this paper as to replicate the first and the second synergy, respectively. The the bottom one shows the configuration with the same stiffness in the all the joints.

Furthermore, we tested the grasping ability of the device with the realized configurations. We selected a representative subset of the objects contained in the YCB grasping toolkit [25] and we asked to a healthy subject to wear the devices with the different stiffness configurations and to try to stably grasp the considered objects. Ten objects were selected so to span different sizes and shapes: meat can, coffee can, strawberry, soft ball, golf ball, apple, metal mug, baseball, gelatin box and mustard container. We restrained the motion of the arm of the subject so to effectively test only adaptability 
of the device to the shape of the object to grasp. The device with the stiffness configuration computed mapping the first synergy was able to stably grasp all the objects The one with the stiffness configuration related to the second synergy was able to grasp six objects (strawberry, baseball, golf ball, apple, gelatin box and mustard container) while, that with the same stiffness values for all the joints only three (strawberry, soft ball, golf ball). As an example, Fig. 11 shows the snapshots with some of the grasped objects with the first mapped synergy configuration.

\section{CONCLUSIONS}

We presented an approach to design the joint stiffness of flexible grippers in order to achieve a desired fingertip flexion trajectory. We explored the role of stiffness in grasping performance and developed a method which is applicable to tendon-driven, underactuated and passively compliant hands. We used the theory of mechanics of tendon-driven hands coupled with beam theory. The feasibility of the approach was demonstrated through a framework composed of both simulations and exploitation of model in the realization of the Soft-SixthFinger. The experiments revealed that modeling joint stiffness and its realization using the proposed method improved the grasping performance and shape adaptation to a wider set of objects. The presented approach can also reduce the manufacturing iterations needed to optimize the joints stiffness.

However, the proposed approach has some limitations. As reported in the experimental section, one limitation is the accuracy of the trajectory tracking which is affected by non modeled factors such as friction and by the approximation of the computed stiffness values to the mean. Other issues are the limited output wrench, the repeatability of finger motion and the durability of the device due to the tendon transmission. Finally, the design of tendon driven fingers that can also perform abduction/adduction motion together with flexionextension is not straightforward.

We are currently investigating these aspects with particular emphasis on the design of fingers with more DoFs. We are also working on the realization of multi-fingered grippers based on the same design principles of the Soft-SixthFinger.

\section{REFERENCES}

[1] S. Hirose and Y. Umetani, "The development of soft gripper for the versatile robot hand," Mechanism and machine theory, vol. 13, no. 3, pp. 351-359, 1978.

[2] L. Birglen, T. Lalibertè, and C. Gosselin, Underactuated Robotic Hands, vol. 40 of Springer Tracts in Advanced Robotics. Springer, 2008.

[3] A. M. Dollar and R. D. Howe, "The highly adaptive sdm hand: Design and performance evaluation," The international journal of robotics research, vol. 29, no. 5, pp. 585-597, 2010.

[4] M. G. Catalano, G. Grioli, E. Farnioli, A. Serio, C. Piazza, and A. Bicchi, "Adaptive synergies for the design and control of the pisa/iit softhand," The International Journal of Robotics Research, vol. 33, no. 5, pp. 768782, 2014.

[5] R. Deimel, C. Eppner, J. Alvarez-Ruiz, M. Maertens, and O. Brock, "Exploitation of environmental constraints in human and robotic grasping," in International Symposium on Robotic Research, 2013.

[6] M. Liarokapis and A. M. Dollar, "Post-contact, in-hand object motion compensation for compliant and underactuated hands," in IEEE International Symposium on Robot and Human Interactive Communication, 2016.
[7] J. Butterfass, M. Grebenstein, H. Liu, and G. Hirzinger, "DLR-hand II: next generation of a dextrous robot hand," in Robotics and Automation, 2001. Proceedings 2001 ICRA. IEEE International Conference on, vol. 1, pp. 109-114, 2001.

[8] F. Sanfilippo, G. Salvietti, H. Zhang, H. P. Hildre, and D. Prattichizzo, "Efficient modular grasping: an iterative approach," in Proc. IEEE Int. Conf. on Biomedical Robotics and Biomechatronics, (Rome, Italy), pp. 1281-1286, 2012.

[9] M. Bächer, S. Coros, and B. Thomaszewski, "Linkedit: interactive linkage editing using symbolic kinematics," ACM Transactions on Graphics (TOG), vol. 34, no. 4, p. 99, 2015.

[10] I. Hussain, G. Salvietti, G. Spagnoletti, and D. Prattichizzo, "The softsixthfinger: a wearable emg controlled robotic extra-finger for grasp compensation in chronic stroke patients," IEEE Robotics and Automation Letters, vol. 1, pp. $1000-1006$, July 2016.

[11] D. Wren and R. B. Fisher, "Dextrous hand grasping strategies using preshapes and digit trajectories," in Systems, Man and Cybernetics, 1995. Intelligent Systems for the 21st Century., IEEE International Conference on, vol. 1, pp. 910-915, IEEE, 1995.

[12] D. G. Kamper, E. G. Cruz, and M. P. Siegel, "Stereotypical fingertip trajectories during grasp," Journal of neurophysiology, vol. 90, no. 6, pp. 3702-3710, 2003.

[13] C. Goldfeder, P. K. Allen, C. Lackner, and R. Pelossof, "Grasp planning via decomposition trees," in Robotics and Automation, 2007 IEEE International Conference on, pp. 4679-4684, IEEE, 2007.

[14] G. Gioioso, G. Salvietti, M. Malvezzi, and D. Prattichizzo, "Mapping synergies from human to robotic hands with dissimilar kinematics: an approach in the object domain," IEEE Trans. on Robotics, 2013.

[15] D. Prattichizzo, M. Malvezzi, M. Gabiccini, and A. Bicchi, "On motion and force controllability of precision grasps with hands actuated by soft synergies," IEEE Transactions on Robotics, vol. in press, pp. 1-17, 2013.

[16] J. Gafford, Y. Ding, A. Harris, T. McKenna, P. Polygerinos, D. Holland, A. Moser, and C. Walsh, "Shape deposition manufacturing of a soft, atraumatic, deployable surgical grasper," Journal of Medical Devices, vol. 8, no. 3, p. 030927, 2014.

[17] D. P. Holland, E. J. Park, P. Polygerinos, G. J. Bennett, and C. J. Walsh, "The soft robotics toolkit: shared resources for research and design," Soft Robotics, vol. 1, no. 3, pp. 224-230, 2014.

[18] L. U. Odhner and A. M. Dollar, "The smooth curvature model: An efficient representation of euler-bernoulli flexures as robot joints," IEEE Transactions on Robotics, vol. 28, no. 4, pp. 761-772, 2012.

[19] J. A. Gopi and G. B. Nando, "Modeling of youngs modulus of thermoplastic polyurethane and polydimethylsiloxane rubber blends based on phase morphology," Advances in Polymer Science and Technology: An International Journal, pp. 43-51, 2014.

[20] C. Hohimer, J. Christ, N. Aliheidari, C. Mo, and A. Ameli, "3d printed thermoplastic polyurethane with isotropic material properties," in SPIE Smart Structures and Materials + Nondestructive Evaluation and Health Monitoring, pp. 1016511-1016511, International Society for Optics and Photonics, 2017.

[21] D. Prattichizzo, G. Salvietti, F. Chinello, and M. Malvezzi, "An objectbased mapping algorithm to control wearable robotic extra-fingers," in Proc. IEEE/ASME Int. Conf. on Advanced Intelligent Mechatronics, (Besanon, France), pp. 1563-1568, 2014.

[22] M. Santello, M. Flanders, and J. F. Soechting, "Postural hand synergies for tool use," The Journal of Neuroscience, vol. 18, pp. 10105-10115, December 1998.

[23] M. Malvezzi, G. Gioioso, G. Salvietti, and D. Prattichizzo, "Syngrasp: A matlab toolbox for underactuated and compliant hands," Robotics Automation Magazine, IEEE, vol. 22, pp. 52-68, December 2015.

[24] M. Skouras, B. Thomaszewski, S. Coros, B. Bickel, and M. Gross, "Computational design of actuated deformable characters," ACM Transactions on Graphics (TOG), vol. 32, no. 4, p. 82, 2013.

[25] B. Çalli, A. Walsman, A. Singh, S. Srinivasa, P. Abbeel, and A. M. Dollar, "Benchmarking in manipulation research: The YCB object and model set and benchmarking protocols," CoRR, vol. abs/1502.03143, 2015 . 